\title{
Are cascading flows stable?
}

\author{
S. A. THORPE ${ }^{1} \dagger$ AND B. OZEN ${ }^{2}$ \\ ${ }^{1}$ School of Ocean Sciences, Marine Science Laboratories, Menai Bridge, Anglesey LL59 5AB, UK \\ ${ }^{2}$ Laboratoire de Recherches Hydrauliques, Ecole Polytechnique Fédérale de Lausanne, CH-1015 \\ Lausanne, Switzerland
}

(Received 28 February 2007 and in revised form 31 May 2007)

The stability of flows cascading down slopes as dense inclined plumes is examined, with particular reference to flows observed in Lake Geneva during winter periods of severe cooling. A previous conjecture by Turner that the flow may be in a state of marginal stability is confirmed: the observed mean velocity and density profiles are unstable to Kelvin-Helmholtz instability, but only marginally so; the growth rates of the most unstable small disturbances to the cascading flow in Lake Geneva are small, with e-folding periods of about $2 \mathrm{~h}$. A reduction in the maximum velocity by about $20 \%$ is required to stabilize the flow.

The possibility that stationary hydraulic jumps may occur in the observed flow is also considered. Several plausible flow states downstream of transitions are examined, allowing for mixing and density changes to occur, ranging from one that preserves the shape of the density and velocity profiles to one in which, as a consequence of mixing, the velocity and density become uniform in depth within the cascading flow. Neither of these extreme states is found to conserve the fluxes of volume, mass and momentum through a transition in which the energy flux does not increase, and to be unique or 'stable' in the sense that no further transition is possible to a similar flow state without more entrainment. Stable transitions to intermediate downstream flows that conserve flow properties and reduce energy flux are, however, found, although the smallest value of the flow parameter, $F r \equiv U_{\max }^{2} / g \Delta h$ (where $U_{\max }$ is the maximum flow speed, $g$ is the acceleration due to gravity, $\Delta$ is a fractional density difference within the flow and $h$ is the flow thickness) at which transitions may occur is only slightly less than that of the cascading flow in Lake Geneva. In this sense, the observed flow is marginally unstable to a finite-amplitude transition or hydraulic jump. Velocity and density profiles of possible flows downstream of a transition are found. The amplitudes of possible transitions and the flux of water entrained from the ambient overlying water mass are limited to narrow ranges.

\section{Introduction}

During very cold winter nights, cold water formed in the shallows around the edges of the Lake Geneva cascades in gravity currents, each flowing for several hours down the sloping sides of the lake and intruding into the upper part of the thermocline (Fer, Lemmin \& Thorpe 2002a). Figure $1(a, c)$ shows profiles of the down-slope component of velocity, $U(z)$, the along-slope component, $V(z)$, and the density, $\rho(z)$, respectively, in the cascading water averaged over a $2 \mathrm{~h}$ period. The example was

\footnotetext{
$\dagger$ Correspondence to 'Bodfryn', Glanrafon, Llangoed, Anglesey LL58 8PH, UK. oss413@sos. bangor.ac.uk.
} 

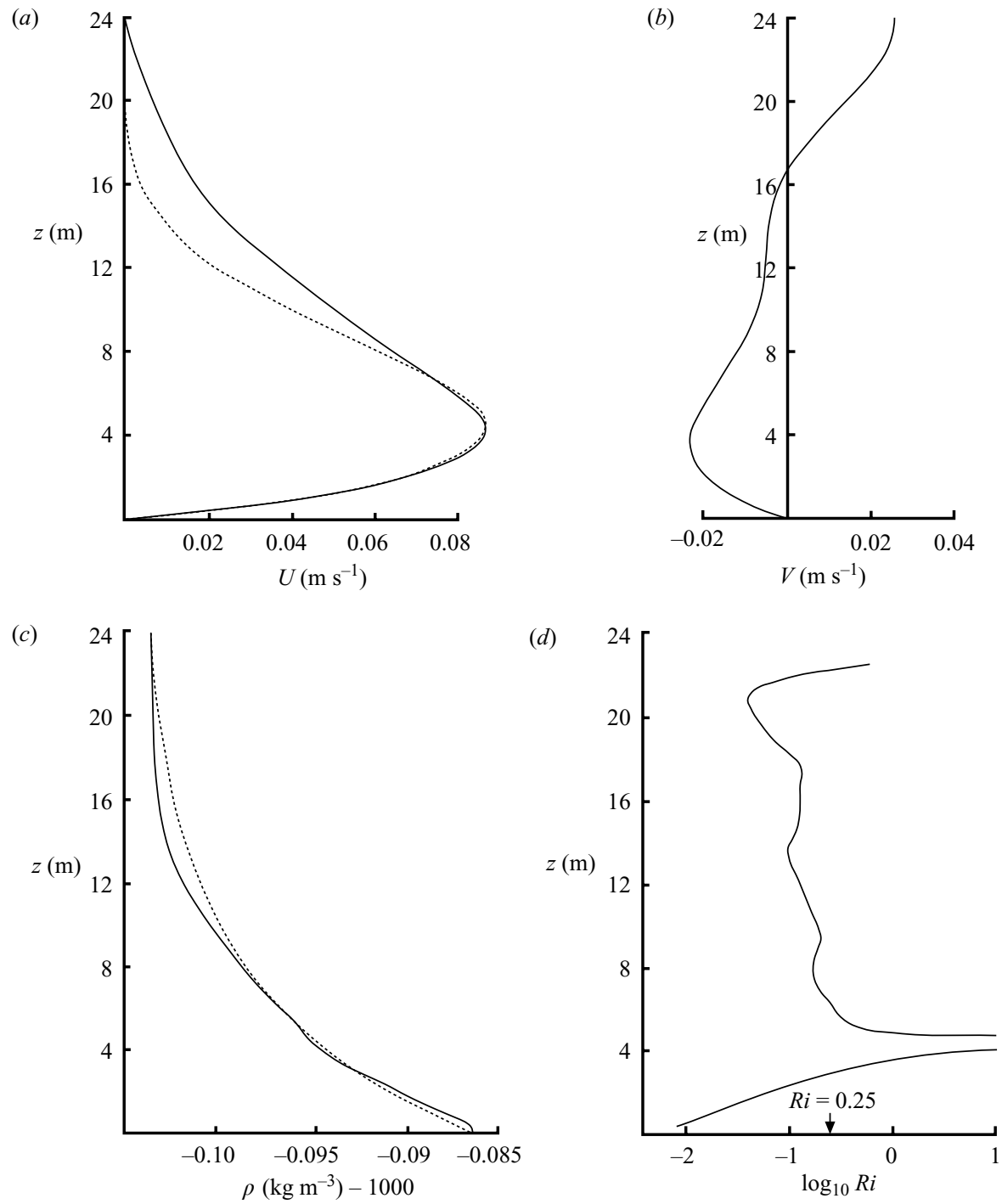

FIGURE 1. Profiles of $(a)$ mean down-slope component of velocity, $U(z),(b)$ mean along-slope component of velocity, $V(z),(c)$ mean density, and $(d)$ gradient Richardson number (estimated over $1 \mathrm{~m}$ and plotted in logarithmic form) obtained from observations in a $2 \mathrm{~h}$ period of relatively steady flow in an inclined plume flowing down a mean slope of about $4.6^{\circ}$ during winter in Lake Geneva. The $V$ component is part of the generally cyclonic wind-driven circulation in the lake modified by internal seiches and by the effects of the Earth's rotation. The dotted curves in $(a)$ and $(c)$ are approximate fits of analytical shapes of velocity (given by (2) with $n=4)$ and density ((3) with $\alpha h=3.5)$ to the observed data, as explained in the text.

selected as one having a relatively small along-slope component of velocity to avoid the complications that such flows may introduce.

Such down-slope flows of dense fluid are of common occurrence (Simpson 1997). The profiles observed in Lake Geneva are similar in form to those of the classic laboratory experiments by Ellison \& Turner (1959) shown in figure 2. The maximum current, $U_{\max }$, is at a height above the bottom, $h_{\max }$, of about 0.2 times the thickness, $h$, of the down-slope flowing layer. (Observations in Lake Geneva find that $h_{m} / h$ is 

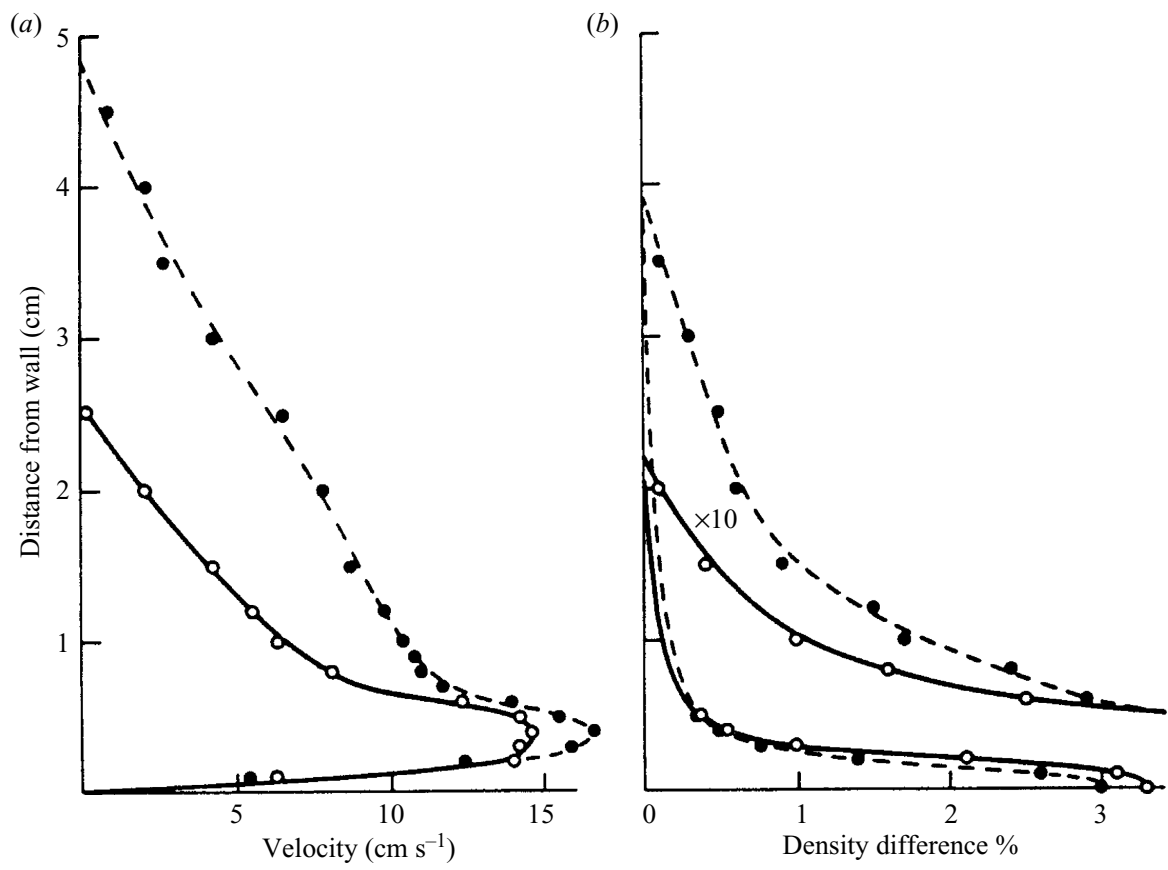

FiguRE 2. Typical profiles of $(a)$ velocity at distances of $\bigcirc, 60$ and $\bullet, 130 \mathrm{~cm}$ from the inlet, and $(b)$ density at distances of $\bigcirc, 70$ and $\bullet, 140 \mathrm{~cm}$ (the upper points being multiplied by a factor of 10 to show variations at small density differences), measured in an inclined plume on a uniform slope of $14^{\circ}$ by Ellison \& Turner (1959) in a laboratory experiment.

$0.18 \pm 0.05$, whereas Ellison \& Turner's two experiments of which profiles are shown in figure 2 give slightly lower values, 0.15 and 0.09). At heights between $h_{\max }$ and $h$ there is a region in which the velocity decays roughly linearly with height. The largest shear is found near the bottom at $z=0$. The density decreases with height, $z$, roughly exponentially, although there is often an almost uniform layer very close to the bottom, perhaps mixed by turbulence generated by the bed shear-stress and, like the velocity, a near-linear decrease in density above the height, $h_{\max }$. In some profiles there is also a shallow region of higher density gradient near $h_{\max }$. The value of $F r \equiv U_{\max }^{2} / g \Delta h$ is used to characterize the flow. Here $\Delta$ is a fractional density difference, the difference between the densities in the flow at the bed and at height, $h$, divided by their sum, and $g$ is the acceleration due to gravity. (The value of $F r$ used here is half that defined using a fractional density difference based on the difference in densities divided by their mean). $\mathrm{Fr}$ is about 3.89 in Ellison \& Turner's experiments on a slope of $14^{\circ}$ and values range from about 2 to 4 in Lake Geneva where the bottom slope is about $4.6^{\circ}$. The value of $\mathrm{Fr}$ corresponding to the flow shown in figure 1 is 3.34. Ellison \& Turner find that a dynamical balance between the frictional and buoyancy forces is achieved in a plume at a down-slope distance from its source of about $10 h_{0}$, where $h_{0}$ is the initial plume thickness. Taking $h_{0}$ as the water depth in the shallow shelf region surrounding the lake, plumes in Lake Geneva should achieve a dynamically balanced state in a down-slope distance of $30-50 \mathrm{~m}$, a distance short compared to the length, several hundred metres, of the distance to the location of the observations in figure 1. 
Values of the gradient Richardson number, $R i$, determined from the mean downslope velocity and density profiles in Lake Geneva are shown in figure $1(d)$. Values less than the Miles-Howard condition necessary for flow stability, 0.25 , are found in the region above the level $z=h_{\max }$ (where $R i$ tends to infinity) and $R i$ is also relatively small near the bed. Turner $(1973, \S 6.2 .4)$ introduces the idea that the upper region of an inclined plume, a region where the flow is turbulent but where the mean velocity and density profiles are approximately linear, could be maintained in a state of marginal stability. It is physically plausible for the mean flow to be maintained in a marginal state because, when turbulence is enhanced by instability of the mean flow, so too is the turbulent Reynolds stress, and so the flow subsequently decelerates, leading to a decay in turbulence. However, the fluid still contains residual stratification, being most dense near the sloping boundary, and so is then accelerated by the down-slope component of gravity, leading to renewed instability with a further increase in turbulence, and the cycle repeats, never developing far from the marginal state at which instability occurs. In support of this concept, Turner $(1973, \S 5.3 .2)$ quotes Mittendorf's (1961) observations of repeated Kelvin-Helmholtz instability in flow in a tilted tube filled with, initially, two distinct miscible layers. Instability in the form of billows is followed by flow deceleration, the collapse of turbulence, and flow acceleration followed by the repetition of instability. (This idea was applied by Thorpe \& Hall (1977) in a study of the stability of a wind-forced stratified near-surface flow in Loch Ness. The mean flow was found to be stable, but close to conditions of marginal stability). From the experiments of Ellison \& Turner (1959), Turner estimates that the gradient Richardson number of the mean flow in the region, assumed to be near the critical Richardson number, $R i_{c}$, characterizing marginal stability, is about $0.062 \pm 0.002$. (Correction has been made for the tilt of isopycnals.) It is remarkable that, although the flow is turbulent, its nature is possibly characterized by a parameter based on the linear stability of an inviscid and steady mean flow. Turner does not, however, establish whether the observed mean flow is close to a marginal state when the Richardson in the region of near-linear profiles is about 0.062 .

The steady cascading flows in Lake Geneva are punctuated by pulses of water, apparently similar to the roll waves that occur in steep and shallow open channel flows (Fer et al. 2002a), and similar pulsations are observed in both rotating and non-rotating flows (Cenedese et al. 2004). No such pulsations or flow variability were observed by Ellison \& Turner (private communication, J. S. Turner). Fluctuations in a developing inclined plume are studied by Pawlak \& Armi (2000) in connection with the observed flows over the sill in Knight Inlet, British Columbia. They attribute the large-scale variations observed in the flow to the marginal stability of the upper shear layer, but do not examine the solutions of the Taylor-Goldstein equation (Drazin \& Reid 1981) to see if this is the case, and disregard the effects on the flow of the bottom boundary layer. They observe that the frequency, measured at a fixed point, of the passing large-scale structures attributed to marginally unstable disturbances in the flow, is approximately equal to the buoyancy frequency in the stratified plume.

Similar flow fluctuations are found in down-slope windstorms in the atmospheric boundary layer in the lee of topographic ridges, although in conditions of more complex flows affected by radiating internal waves. Neiman et al. (1988), for example, report fluctuations at two frequencies in cascading flow in the foothills west of Boulder, Colorado. Peltier \& Scinocca (1990) solve the Taylor-Goldstein equation and the corresponding equation describing the spatial growth of disturbances, and demonstrate that, in accord with analysis by Gaster (1962) growth rates are equivalent. (Gaster (1962) demonstrates that the temporal growth rate, $\sigma_{i}$, and the spatial growth 
rate, $k_{i}$, are related by the disturbance group velocity, $\partial \sigma_{r} / \partial k_{r}: \sigma_{i} / k_{i}=-\partial \sigma_{r} / \partial k_{r}$, where subscripts $r$ and $i$ represent real and imaginary parts of the frequency, $\sigma$, and wavenumber, $k$, of the disturbance.) Peltier \& Scinocca attribute the fluctuations observed at the ground to Kelvin-Helmholtz instability centred in a shear layer at a height of some $4 \mathrm{~km}$.

Our objective is to examine the stability of cascading flows to small disturbances taking as a particular example the flow observed in Lake Geneva and, further, to establish the conditions in which hydraulic jumps or transitions to different flows may occur. The former study provides information to address whether the flow is in a state of marginal stability as suggested by Turner, whilst the latter is related to the stability of the flow in passing finite changes in bottom topography or, as in a flow downstream of a weir, as the water cascading down a slope enters a pool of denser water, the lake's thermocline. Although the steady but turbulent down-slope flows that are observed are a consequence of a balance between the down-slope component of gravitational forces and the combined drag forces of the bottom and overlying fluid acting on the current, for simplicity we shall neglect the effects of friction and turbulence on the mean flow and disregard the bottom slope in the way that is common in studies of hydraulic phenomena in open-channel flows. We shall therefore ignore the component of gravity parallel to the sloping boundary and, in the subsequent analysis of Kelvin-Helmholtz instability and hydraulic transitions, we consider the stability of steady laminar inviscid flows over a horizontal boundary, choosing some analytical forms for the velocity and density profiles of the inclined plumes as well as those shown in figures 1 and 2, so as to investigate the sensitivity of our conclusions to the flow conditions.

We first consider the possibility of Kelvin-Helmholtz instability in some analytical velocity and density profiles resembling the flows observed (\$§ 2.1-2.2). Readers interested in the observed flows may wish to omit $\S 2.2$ and go to the discussion of their stability in $\S 2.3$. The results show that the flows are marginally unstable as conjectured by Turner, even though the minimum gradient Richardson numbers are substantially less than the commonly adopted (or assumed) 'critical' value of 0.25 (e.g. see Gray, Alexander \& Leeder 2006). The conditions for transitions or hydraulic jumps to occur from the observed flows to others are considered in $\S 3$. These conditions, characterized by the size of $\mathrm{Fr}$ and the amplitude of jumps, naturally depend on the types of flow selected downstream of the transition. For the flow observed in Lake Geneva, the downstream flow states, the velocity and density profiles to which transitions are possible, and the amplitude of the jumps, appear to be limited. The main results are discussed in $\S 4$.

\section{Kelvin-Helmholtz instability}

\subsection{Known solutions for boundary jet profiles}

The stability of an inviscid stratified shear flow, $U(z)$, to small disturbances is determined by the solutions of the Taylor-Goldstein equation,

$$
\mathrm{d}^{2} \varphi / \mathrm{d} z^{2}+\left\{N^{2} /(U-c)^{2}-k^{2}-\mathrm{d}^{2} U / \mathrm{d} z^{2} /(U-c)\right\} \varphi=0,
$$

where the streamfunction is $\psi(x, z, t)=\phi(z) \exp [\mathrm{i} k(x-c t)], k$ is the (real) wavenumber in the horizontal $x$-direction, $c=c_{r}+\mathrm{i} c_{i}$ is the complex wave phase speed and $N$ is the buoyancy frequency. Boundary conditions are that the vertical velocity, and therefore $\phi(z)$, are zero at the rigid boundary, $z=0$, and that $\phi(z)$ tends to zero as $z$ 
tends to infinity. Solutions with $k c_{i}>0$ grow exponentially in time and are therefore unstable.

It may be anticipated that the critical Richardson number, $R i_{c}$, in stably stratified jet-like flows over a rigid boundary will be less than 0.25. Miles (1967) showed that flows in which the velocity and density both decrease exponentially at the same rate with distance from a horizontal boundary are stable at all positive values of Richardson number; $R i_{c}$ is equal to zero. Profiles with $\mathrm{d} U / \mathrm{d} z$ and $\mathrm{d} \rho / \mathrm{d} z$ constant in a layer adjoining a horizontal boundary, a layer above which the velocity and density are constant, are also found to be stable, with $R i_{c}=0$.

\subsection{Some numerical solutions of analytical boundary jet profiles}

Hazel (1972) solves the Taylor-Goldstein equation numerically and finds that, when $N$ is constant, the critical Richardson number, $R i_{c}$, of second mode disturbance of the Bickley jet $\left(U(z)=U_{1} \operatorname{sech}^{2} a z\right)$ is 0.184 . The central streamline (at $\left.z=0\right)$ to the second mode disturbances is horizontal and may be replaced by a rigid horizontal plane; the second mode solution of (1) is identical to that of a jet-like flow over a rigid boundary. As before, $R i_{c}<0.25$. The resulting 'half Bickley jet' profile differs from the examples in $\S 2.1$ in that it contains a velocity inflection point removed from the horizontal plane at $z=0$. Inflection points, although not essential for instability in stratified flows, are known by Rayleigh's inflection point theorem to be necessary for instability in unstratified flows and may help promote instability in stratified flows.

None of the above examples, however, satisfies a no-slip condition at the horizontal boundary: unlike the profiles shown in figure 1 and 2 , the mean velocity does not tend to zero at $z=0$. Some analytic profiles that satisfy the no-slip condition of the mean flow are considered to provide illustration and reference before the real flows are examined in the next section.

A form of velocity profile that reproduces some of the main features that are observed is

$$
U(z)=U_{1}[\pi(1-z / h)]^{n} \sin [\pi(1-z / h)],
$$

where $U_{1}$ is a constant velocity, $h$ is the thickness of the layer moving down-slope, and $n$ is an integer. Figure 3(a) shows the velocity profiles for selected values of $n$. The value $n=4$ gives a velocity profile that has a maximum, $U_{\max }$, equal to $23.61 U_{1}$, at $h_{\max } / h=0.183$, corresponding approximately to the height of the velocity maximum in the velocity profile of figure $1(a)$, where (with speeds, $U_{\max }$, matched) it is shown for comparison by a dotted line. It underestimates the velocity gradient in the region above $h_{\max }$. There is an inflection point in the $n=4$ velocity profile at $z=h_{\text {inf }}$ where $h_{\text {inf }} / h=0.355$ and where $U=15.1 U_{1}$ or $0.64 U_{\max }$. Some of the properties of this and other profiles are given in table 1 .

An exponential density profile,

$$
\rho(z)=\rho_{0}\{1-\Delta+2 \Delta([\exp (-\alpha z)-\exp (-\alpha h)] /[1-\exp (-\alpha h)])\},
$$

where $\rho_{0}$ is a reference density and $\Delta$ is a non-dimensional constant, so that $\rho(h)=\rho_{0}[1-\Delta]$ and $\rho(0)=\rho_{0}[1+\Delta]$, is an approximate fit to the observed profile in Lake Geneva (figure $1 c$ ) when $\alpha h \approx 3.5$. It is shown by curve A in figure $3(b)$ and by the dotted line fitted to the observed density range in figure $1(c)$. The gradient Richardson number, $R i=-\left(g / \rho_{0}\right) \mathrm{d} \rho / \mathrm{d} z /(\mathrm{d} U / \mathrm{d} z)^{2}$, for the velocity profile (2) with $n=4$ and when the density is represented by (3) with $\alpha h=3.5$, is shown in logarithmic form in figure $3(c)$. As explained later, it is scaled so that the minimum $R i$ has the value found at marginal stability. The Richardson number is infinite at the velocity maximum, $z / h=0.183$, and has its smallest value, $R i_{0}$, at the boundary, $z=0$. It has 
(a)

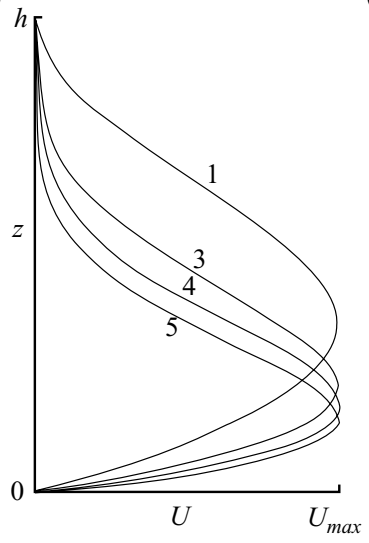

(b)

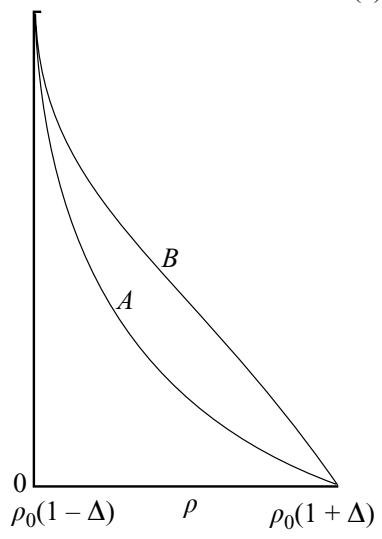

(c)

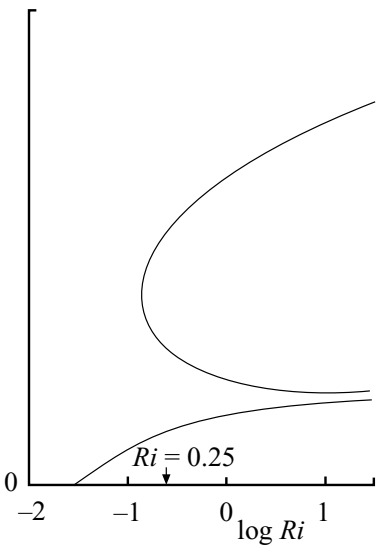

Figure 3. Profiles of (a) velocity given by (2) for various specified values of $n$, and $(b)$ density given by A: (3) with $\alpha h=3.5$ and $\mathrm{B}:(4)$. (c) shows the logarithm of the Richardson number, $\log R i$, corresponding to the velocity profile in $(a)$ with $n=4$ and the density profile A of $(b)$. The Richardson number is scaled to fit the conditions of marginal stability with $R i_{0}=0.028$.

$\begin{array}{cccccccc}U(z) & \rho(z) & U_{\max } / U_{1} & W / U_{\max } & H / h_{1} & R i_{\text {inf }} / R i_{0} & h_{\max } / h & h_{\text {inf }} / h \\ (2), n=3 & (3), \alpha h=3.5 & 9.37 & 0.744 & 0.555 & 3.30 & 0.220 & 0.423 \\ (2), n=4 & (3), \alpha h=3.5 & 23.6 & 0.736 & 0.494 & 5.42 & 0.183 & 0.355 \\ (2), n=5 & (3), \alpha h=3.5 & 61.7 & 0.729 & 0.445 & 7.74 & 0.155 & 0.305 \\ (2), n=4 & (3), \alpha h=2.5 & 23.6 & 0.744 & 0.494 & 7.73 & 0.183 & 0.355 \\ (2), n=4 & (3), \alpha h=4.5 & 23.6 & 0.744 & 0.494 & 3.79 & 0.183 & 0.355 \\ (2), n=4 & (4) & 23.6 & 0.744 & 0.494 & 26.0 & 0.183 & 0.355 \\ \text { Figure 1(a) } & \text { Figure 1(c) } & 1 & 0.697 & 0.633 & 16.3 & 0.187 & 0.304\end{array}$

TABLE 1. Characteristic values of the model and observed flows used to study the onset of Kelvin-Helmholtz instability. Values are given of ratios characterizing 6 models of a stratified boundary jet of depth, $h$, defined by equations (2), (3) and (4) as listed under $U(z)$ and $\rho(z)$, and from the profiles of the observed cascading flows in Lake Geneva, figure. 1 $(a-c)$, respectively. $U_{\max }$ is the maximum current, found at height $h_{\max }$, and $W$ and $H$ are integral scales given by $W H=\int U(z) \mathrm{d} z$ and $W^{2} H=\int U^{2}(z) \mathrm{d} z$. The Richardson numbers are $R i_{\text {inf }}$ and $R i_{0}$ at the inflection point at height, $h_{\text {inf }}$, in the velocity profile and at the bottom, $z=0$, respectively. There is considerable uncertainty in the estimate of $R i_{\text {inf }} / R i_{0}$ for the observed profile.

a second, but larger, minimum, at $z / h=0.405$ where $R i$ is a factor of 5.03 greater than $R i_{0}$. The Richardson number at the inflection point at $z / h=0.355, R i_{\text {inf }}$, a useful measure of $R i$ in the layer above the velocity maximum, is $5.42 R i_{0}$. (This is as shown in table 1.) The minimum $R i$ can be written as $R i_{0}=0.0430 g \Delta h / U_{\max }^{2}$ and so $R i_{\text {inf }}=0.233 g \Delta h / U_{\max }^{2}$ or $0.233 \mathrm{Fr}^{-1}$. In recognition of its significance in the Miles-Howard theorem, it is usual to use the minimum value of $R i$ (here that at $z=0$ ) in defining a critical value, but, in this case, values are more easily measured above the velocity maximum, in particular at the inflection point in the velocity profile, and we therefore refer to both, e.g. in table 2.

The equivalence between the growth of temporally growing and spatially growing disturbances with low growth rates in such stratified shear flows is established by Peltier \& Scinocca (1990). We have therefore solved the Taylor-Goldstein equation using the profiles of velocity and density, (2) and (3), in $0 \leqslant z \leqslant h$ and with $U(z)=0$ 


\begin{tabular}{ccccccc}
\hline$U(z)$ & $\rho(z)$ & $R i_{c}$ & $k h$ & $F r_{c}$ & $R i_{\text {infc }}$ & $F_{c}$ \\
$(2), n=3$ & $(3), \alpha h=3.5$ & 0.045 & 1.7 & 1.49 & 0.149 & 1.49 \\
$(2), n=4$ & $(3), \alpha h=3.5$ & 0.028 & 1.8 & 1.54 & 0.151 & 1.68 \\
$(2), n=5$ & $(3), \alpha h=3.5$ & 0.020 & 1.95 & 1.49 & 0.155 & 1.78 \\
$(2), n=4$ & $(3), \alpha h=2.5$ & 0.020 & 1.85 & 1.62 & 0.155 & 1.77 \\
$(2), n=4$ & $(3), \alpha h=4.5$ & 0.039 & 1.9 & 1.39 & 0.148 & 1.52 \\
$(2), n=4$ & $(4)$ & 0.0059 & 1.9 & 2.02 & 0.153 & 2.21 \\
Figuge 1(a) & Figure 1(c) & $0.0035 \pm 0.0002$ & 1.8 & 2.23 & 0.24 & 1.32
\end{tabular}

TABLE 2. Critical values at the onset of Kelvin-Helmholtz instability. As in table 1, values are given from 6 models of a stratified boundary jet of depth, $h$, and from the profiles of the observed cascading flows in figure $1(a-c)$. The value of the critical Richardson number, $R i_{c}$, is the minimum value of $R i$ in the profiles at the onset of instability, in each case being that at height, $z=0$. The non-dimensional wavenumber, $k h$, is that of the disturbance wavenumber that first becomes unstable and is in each case close to the fastest growing disturbances when the minimum $R i$ is less than $R i_{c}$. The term, $F_{c}=U_{\max }^{2} / g \Delta h$, is the smallest value at which instability can occur. The critical Richardson number at the inflection point in the velocity profile is $R i_{\text {infc }}$ and $F_{c}$ is the value of the non-dimensional number, $W^{2} / g \Delta H$, based on the integral scales, $W$ and $H$, at marginal stability.

(a)

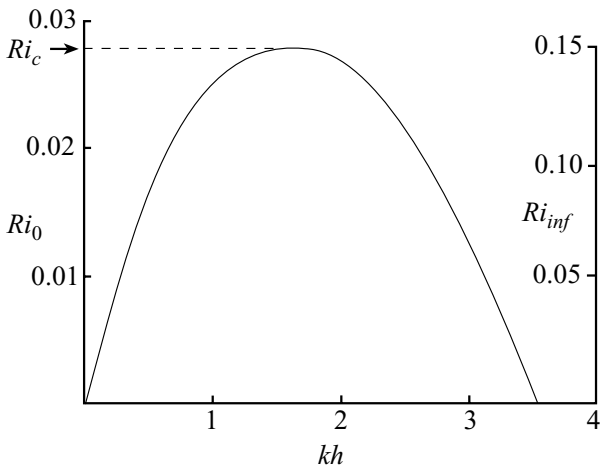

(b)

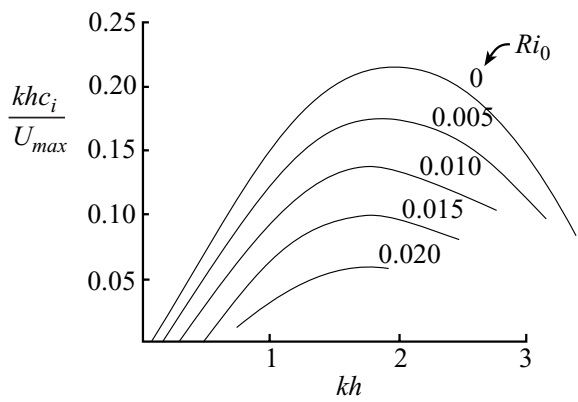

FIGURE 4. Stability curves for first mode disturbances in a flow with velocity given by (2) with $n=4$ and density by (3) with $\alpha h=3.5$. (a) the neutral curve in the plane of $R i_{0}$ (the value of $R i$ at $z=0)$ and $R i_{\text {inf }}$ versus $k h$ and, $(b)$, the growth rates, non-dimensionalized by dividing the dimensional rates by $U_{\max } / h$, as a function of $k h$ at different specified values of $R i_{0}$. (Corresponding values of $R i_{\text {inf }}$ can be found by multiplying by 5.42.)

and $\rho(z)=\rho_{0}[1-\Delta]$ in $z>h$, so that $U$ and $\rho$ are continuous at $z=h$. Numerical solutions of (1) are obtained using a standard 'shooting' method over a range of possible values of the real phase speed $\left(0<c_{r}<U_{\max }\right)$ and non-zero imaginary phase speeds, $c_{i}$, with corresponding growth rates, $k c_{i}$, seeking conditions in which the vertical velocities and pressures in the streamfunction solutions within the stratified shear layer at $z=h$ are matched to potential flows in the uniform overlying layers in $z>h$, and with interpolation to find the stability boundaries. Details of the matching conditions are given by Drazin \& Reid $(1981, \S 23)$. Integration through the stratified shear layer makes use of Stoermer's rule (see Press et al. 1992, $\$ 16.5$ ). The number of turning points of the streamfunction, giving the mode of the disturbances, is counted and the solutions constrained to the mode number under investigation.

Figure $4(a)$ shows the stability curve in the plane of $R i_{0}$ (the value of $R i$ at $z=0$ ) or $R i_{\text {inf }}$ versus non-dimensional wavenumber, $k h$, for the forms of velocity given by 
(2) when $n=4$ and density by (3) with $\alpha h=3.5$. Growth rates for the first mode, non-dimensionalized with $U_{\max } / h$, are shown in figure $4(b)$. No modes higher than the first are found to be unstable. The critical Richardson number, $R i_{c}$, the largest value of $R i_{0}$ at which instability can occur, is 0.028 , and the profile of $\log (R i)$ when $R i_{0}=0.028$, the marginal value, is that shown in figure $3(c)$. The equivalent condition for instability of the flow becomes $F r \equiv U_{\max }^{2} / g \Delta h>1.54,=F r_{c}$, where $F r_{c}$ represents the critical value above which flow is unstable. The value of $R i_{\text {inf }}$ at marginal stability, $R i_{\text {infc }}$, is 0.151 . As foreseen, the critical Richardson number is therefore significantly less than 0.25 (indicated by an arrow in figure $3 c$ ) and the minimum $R i$ in the flow above the velocity maximum is 0.14 . (This is substantially greater than the value of about $0.062 \pm 0.002$ observed in the upper region of inclined plumes by Ellison \& Turner and, although the profiles of velocity and density differ, suggests that flows less than those observed by Ellison \& Turner by a factor 1.5 may be 'just unstable'.)

As shown in figure $4(b)$, the growth rates of disturbances at a given value of $k h$ increase as $R i_{0}$ decreases. The greatest dimensional exponential growth rate is $0.217 U_{\max } / h$ in the absence of stratification (or when $R i_{0}=0$ ) and when $k h=1.95$. The maximum growth rates depend on $R i_{0}$ but are close to the non-dimensional wavenumber, $k h=1.8$, corresponding to a wavelength, $2 \pi / k$, equal to $3.49 h$. For example, from figure $4(b)$, the maximum growth rate of disturbances when $R i_{0}=0.012$ (or $R i_{\text {inf }}=0.065$ ) is about $0.12 U_{\text {max }} / h$.

At constant values of $R i<R i_{c}$, the phase speed of unstable disturbances and the height at which their streamfunctions, and therefore the vertical velocities, is a maximum, both decrease with non-dimensional wavenumber $k h$. The streamfunction, and therefore the vertical velocity of the fastest growing waves, has a maximum at about $z / h=0.58$, well above the level of the inflection point, $z / h=0.355$. The phase speed of the fastest growing waves is, however, close to the speed, $0.64 U_{\max }$, at the inflection point. This implies that the frequency of the fastest growing disturbances, $\sigma_{r}$, measured at a fixed point is about $k U$, where $U$ is the flow speed at the inflection point, giving a frequency, $\sigma_{r}=0.64 \times 1.8 U_{\max } / h=1.15 U_{\max } / h$. The buoyancy frequency at the inflection point, $N_{\text {inf }}$, is found, using (3), to be given by $\left.N_{\text {inf }}^{2}=2(g \Delta / h)(\alpha h) \exp [-(\alpha h)(z / h)] /[1-\exp (-\alpha h)]\right\} \quad$ at $z / h=0.355$, or, with $\alpha h=3.5, N_{\text {inf }}=1.44(\mathrm{~g} \Delta / h)^{1 / 2}$. It follows that $N_{\text {inf }} / \sigma_{r}=1.25 \mathrm{Fr}^{-1 / 2}=1.01$ when $F r=U_{\max }^{2} / g \Delta h$ has the marginal value, 1.54. This is consistent with the conclusion by Pawlak \& Armi (2000) that the frequency of disturbances is close to the buoyancy frequency in the shear zone in the upper region of the inclined plume.

For comparison, critical values of parameters for other velocity and density profiles are given in tables 1 and 2 . These include

$$
\rho(z)=\rho_{0}\{1-\Delta+2 \Delta(1-z / h) \sin [(\pi / 2)(1-z / h)]\},
$$

shown in figure 3(b) representing the reduced density gradient near $z=0$ observed in figure 2 (although extending too far from the boundary). In each case, the minimum Richardson number is at $z=0$. Values of $F r_{c}$ range from 1.39 to 2.02, less than the values of $F r=3.34$ and 3.89 observed in Lake Geneva and by Ellison \& Turner in the laboratory, respectively, suggesting that these flows are likely to be unstable. Although $R i_{c}$ is sensitive to the choice of velocity and density profiles, especially those near $z=0$, the wavelengths of the fastest growing disturbances, $2 \pi / k=2 \pi h /(1.7$ to 1.95$)$ have a relatively small range and, scaled to the Lake Geneva and laboratory values of $h$, are about $80 \mathrm{~m}$ and $16 \mathrm{~cm}$, respectively. 


\subsection{Stability of observed profiles}

\subsubsection{The down-slope flow $U(z)$}

Although providing instructive and useful guidance to the likely ranges of $R i_{c}$ and $F r_{c}$, to the wavenumbers of the fastest growing disturbances, and to the sensitivity to the adopted profiles of velocity and density, the analytical profiles analysed in $\S 2.2$ do not precisely represent the observed profiles shown in figures 1 and 2 , and the Taylor-Goldstein equation has therefore been solved numerically with the observed profiles of velocity and density in the range $0 \leqslant z \leqslant h$.

The down-slope flow, $U(z)$, in Lake Geneva is found to be unstable to a disturbance of the first mode. The fastest growing wavelengths are $85.7 \pm 2.3 \mathrm{~m}(k h=1.76 \pm 0.05)$ with phase speeds of $0.0456 \mathrm{~m} \mathrm{~s}^{-1}$ (compared to the maximum current of $0.0865 \mathrm{~m} \mathrm{~s}^{-1}$ ). The maximum amplitude of the vertical velocity of the fastest growing disturbances is greatest at a height of about $15.2 \mathrm{~m}$. The exponential growth rates of these disturbances are, however, very small, $1.365 \times 10^{-4} \mathrm{~s}^{-1}$, corresponding to waves being amplified by a factor $e=2.718$ over a period of $2.04 \mathrm{~h}$. Observed values of $R i$ are a factor $1.5 \pm 0.1$ smaller than critical (characterized either by the minimum $R i$ or by that at the inflection point). The critical value, $F r_{c}$, is 2.23 , compared to the observed value, 3.34 . A reduction in the flow speed of about $20 \%$ is required to stabilize the flow. Flows with the observed shape of profiles will be stable if the Richardson number in the uniform shear region above the velocity maximum exceeds about 0.2 .

In the sense that the growth rates of the fastest growing disturbances are small and that a relatively small decrease in the maximum current would lead to stability, the observed flow in the inclined plume is in a state of marginal instability, as conjectured by Turner.

The speed of propagation of non-growing waves relates to the existence of hydraulic jumps, as discussed in $\S 4$. Solutions of the Taylor-Goldstein equation, (1), are found for upstream propagating waves, those with negative phase speeds, $c_{r}<0$, and with $c_{i}=0$ (non-growing waves) when the density and velocity profiles are chosen as those in Lake Geneva. The phase speed of long mode 1 waves approaches the minimum flow speed (zero in the upper layer and at $z=0$ ) as $F r$ increases, being $-1.39 \times 10^{-2} \mathrm{~m} \mathrm{~s}^{-1}$ when $\mathrm{Fr}=0.209,-3.77 \times 10^{-3} \mathrm{~m} \mathrm{~s}^{-1}$ at the observed value $F r=3.34$, and $-1.69 \times 10^{-3} \mathrm{~m} \mathrm{~s}^{-1}$ at $F r=16.2$. (The phase speed is approximately given by $c=-0.132 U_{\max } F^{-1}$. The minimum $R i$ is less than 0.25 in flows with $\mathrm{Fr}>0.374$, beyond the range of Bell's (1974) general results regarding the properties of internal wave propagation with $R i>0.25$. Upstream wave propagation is in accord with the conclusions of Pratt et al. 2000.)

Solutions of the Taylor-Goldstein equation have also been found for Ellison \& Turner's velocity and density profiles of figure 2 measured at $130 \mathrm{~cm}$ and $140 \mathrm{~cm}$, respectively, from the inlet. The flow is again found to be unstable. Mode 1 disturbances of wavelength $23.3 \mathrm{~cm}$ have a growth rate of $0.362 \mathrm{~s}^{-1}$, so growing exponentially in about $2.76 \mathrm{~s}$. The maximum amplitude of the vertical velocity of this disturbance is greatest at a height of about $0.85 \mathrm{~cm}$ and above the velocity maximum $0.38 \mathrm{~cm}$ above the boundary. The height of the velocity maximum is small and it is doubtful whether neglect of the effects of viscosity is justified in this case.

\subsubsection{The effect of along-slope flow}

The along-slope flow, $V(z)$, (figure $1 b$ ) in Lake Geneva is non-zero and the flow direction varies with $z$. We have therefore examined the stability of the flow $(U(z)$, $V(z))$, to two-dimensional disturbances in directions inclined at angles, $\alpha$, to the 
down-slope direction. As in Thorpe (1999), $U(z)$ in the Taylor-Goldstein equation, (1), is now replaced by $W(z)=U(z) \cos \alpha+V(z) \sin \alpha$, and the streamfunction becomes $\psi(x, z, t)=\phi(z) \exp [\mathrm{i} k(x-c t)]$, where $x$ is now in the direction $\alpha$. This equation has been solved numerically as before to find the maximum growth rate, $k c_{i}$, as a function of $\alpha$. The maximum growth rate is found when $\alpha=3.5^{\circ}$ and $k h=1.81 \pm 0.05$, and differs insignificantly from that in the preceding section for the down-slope flow (when $\alpha=0^{\circ}$.

In summary, in this case at least, the effect of the along-slope flow on the growth rate, wavelength or form of the fastest growing disturbance is very small. The observed flow is marginally unstable to Kelvin-Helmholtz instability.

\section{Hydraulic jumps}

\subsection{Introduction}

We now consider whether steady flows like those observed in cascades can undergo stationary (or 'standing') transitions to other steady flows, a form of finite-amplitude instability. It is assumed that the fluxes of volume, mass and momentum of flows are conserved in passing through a transition, but that the energy flux cannot increase. The presence and effect of turbulence within the flow upstream of a transition is ignored and so, for example, the horizontal components of the Reynolds stress and the flux of turbulent energy into a transition or hydraulic jump are disregarded.

A major problem in the analysis is that, although the flow upstream of a jump is known, or can at least be specified, the flow downstream is unknown. In the past, this problem has generally been avoided by taking two-layer flows with uniform densities and velocities in the layers both up- and down-stream of a jump, and with the density in each layer remaining unchanged through the transition (e.g. see Baines 1995) or changing in one of the two layers as a consequence of entrainment at the jump (Holland et al., 2002). Even if the flow approaching a transition is layered (with Kelvin-Helmholtz instability at the interfaces between layers somehow suppressed), in miscible fluids, a jump to another layered flow with discontinuous density and velocities is unrealistic. In the conditions encountered in real flows, mixing occurs in the transition and the downstream density profile cannot retain a form with discrete uniform layers. The flow considered here does not have a uniform layer structure upstream of a postulated transition, and possibly not downstream. To examine and span the possible range of transitions we shall later make choices of the form of the flow downstream of the transition, selecting extreme examples that span possible cases, including one in which the moving fluid adjoining the rigid boundary becomes uniform in velocity and density downstream of a transition.

It is supposed that fluid may be entrained from the upper layer at a rate, $Q$, within the transition, with a concomitant change in density, but (as justified by Thorpe 2007) the entrained fluid is supposed to carry a negligible flux of kinetic energy into the transition region from the overlying fluid. $\dagger$ A parameter, $P$, is defined so that the ratio of $Q$ to the volume flux ahead of a transition is equal to $P-1(\geqslant 0)$.

\subsection{Transitions from the observed profiles}

We consider possible transitions of the observed down-slope flow, $U(z)$, in Lake Geneva, for simplicity (as in $\S 2.3 .1$ ) at present disregarding the relatively small

$\dagger$ Although not generally significant, a reviewer points out that the flux may affect the location of the curves, $E=0$, in figures 5 and 6 . 
along-slope, $V$, component, as well as the effects of the bottom slope, flow acceleration and rotation.

We first assume, for generality, that the profiles of velocity and density given by the equations,

$$
U(z)=U_{i} F_{i}\left(z / h_{i}\right) \text { when } 0 \leqslant z \leqslant h_{i},
$$

with $U_{i}(z)=0$ when $z>h_{i}$, and

$$
\rho_{i}(z)=\rho_{0}\left[1-\Delta+2 \Delta f_{i}\left(z / h_{i}\right)\right] \text { when } 0 \leqslant z \leqslant h_{i},
$$

with $\rho_{i}(z)=\rho_{0}(1-\Delta)$ when $z>h_{i}$, where $i=1$ represents the steady flow approaching a jump and $i=2$ the flow downstream. Here, $\mathrm{d} f_{i}\left(z / h_{i}\right) / \mathrm{d} z<0$ to ensure static stability, and $f_{1}(0)=1$, so defining $2 \rho_{0} \Delta$ as the density change from $z=0$ to $z=h_{1}$ upstream of a transition. A no-slip condition at the lower boundary is satisfied if $F_{i}(0)=0$. If $F_{i}(1)=0$, the velocity is continuous at $z=h_{i}$.

\subsubsection{The shape-preserving transition}

Conservation equations are derived by Thorpe (2007) and, for easy reference, are summarized in the Appendix. We first suppose that the shape of the profiles of velocity and density are preserved in the transition (a 'shape-preserving transition'), but that the density may decrease, say,

$$
F_{1}\left(z / h_{1}\right)=F_{2}\left(z / h_{2}\right)=F(x)
$$

and

$$
f_{1}\left(z / h_{1}\right)=f(x), \quad f_{2}\left(z / h_{2}\right)=\delta f(x)
$$

where $0<\delta<1$. Taking $U_{i}$ to be the maximum velocity in the velocity profiles, so that $U_{1}=U_{\max }$, and $\left|F_{i}\right| \leqslant 1$, and calculating integrals from the observed profiles of figure $1(a, c)$, we find that (A6) reduces to $P=1 / \delta \geqslant 1$. The integrals in (A9) and (A10) can be found from the values in figure $1(a, c)$, giving

$$
F r=0.2644 q\left(q^{2}-P\right) /\left[P\left(q-P^{2}\right)\right],
$$

and

$$
E^{\prime} /\left(g \rho_{0} \Delta U_{\max } h_{1}^{2}\right)=0.12343 F r\left(1-P^{3} / q^{2}\right)-0.2798 q P+0.00116 q+0.2786,
$$

where $E^{\prime}$ is the reduction in energy flux per unit width through the transition. We define a non-dimensional energy loss rate per unit channel width as

$$
E \equiv E^{\prime} /\left[\left(g \Delta h_{1}\right)^{3 / 2} \rho_{0} h_{1}\right]=\left[E^{\prime} /\left(g \rho_{0} \Delta U_{\max } h_{1}^{2}\right)\right] F r^{1 / 2},
$$

where, as before, $F r=U_{\max }^{2} / g \Delta h_{1}$, and $q=h_{2} / h_{1}$, the ratio of the thickness of the flows downstream and upstream of the jump, and a measure of the jump amplitude. Both $q$ and $P$ are greater than or equal to 1 .

Equations (8) and (9) can be used to determine values of $F r$ and $E$ for a range of values of $P$ and $q, \geqslant 1$, as shown in figure 5(a) together with the contour, $S=1$, given by (A12) that determines whether the flows downstream of transitions at particular values of $P$ and $q$ may themselves be subject to further shape-preserving transitions without entraining fluid from the upper layer: no such transitions can occur when $S<1$ and in such cases the possible transitions are regarded as being stable. No real values of $U_{\max }$ are possible in regions where $\mathrm{Fr}<0$. The figure shows that $E<0$ for all values of $P$ and $q$ on the curve $F r=3.34$. No shape-preserving transitions are therefore possible for the flow with $F r=3.34$ observed in Lake Geneva. Indeed, for flows with profiles of velocity and density with the observed shape, none can occur 

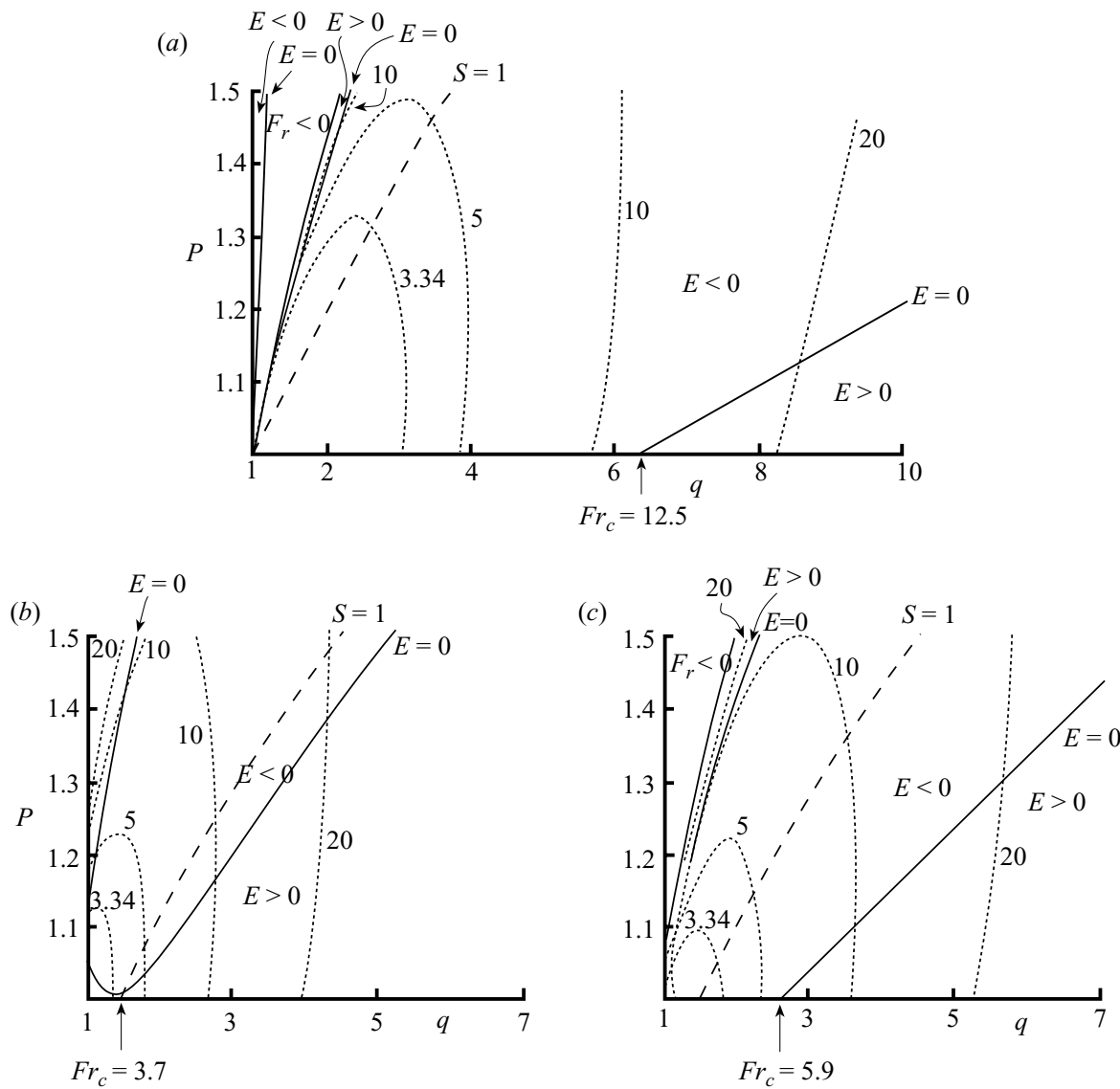

FIGURE 5. The curves, $E=0$, of zero loss in energy flux and of, $S=1$, bounding regions of stable transitions as $q$ and $P$ vary. For given $P, S<1$ and the flow is stable at values of $q$ exceeding those on $S=1$, i.e. to the right-hand side of the curve $S=1$. The flow profiles upstream of a possible transition are as shown in figure $1(a, c)$. The cases illustrated are $(a)$ the shape-preserving transition; $(b)$ the transition to mixed conditions of uniform velocity and density; $(c)$ the transitions to flows with linear density profiles and velocity profiles (2) with $n=1$. Regions $E>0$ and $E<0$ are labelled. The line $S=1$ is shown as a dashed line and contours of $F r=U_{\max }^{2} / g \Delta h_{1}$ are shown by labelled dotted lines. The limiting values of $F r\left(F r_{c}\right)$ at $E=0$ or $S=1$ are indicated by arrows. The value of $F r$ observed in the Lake Geneva data is 3.34 , one of the contoured curves.

until $\mathrm{Fr}$ exceeds 12.5 when only jumps of amplitude $q>6.36$ conserve volume, mass and momentum fluxes and lose energy in the transition. It is therefore necessary to increase $U_{\max }$ by a factor 1.93 from $0.0865 \mathrm{~m} \mathrm{~s}^{-1}$ to about $0.167 \mathrm{~m} \mathrm{~s}^{-1}$, keeping $h_{1}$ and $\Delta$ constant, before a jump preserving the profile shapes can occur.

A similar analysis of the profiles of velocity at $130 \mathrm{~cm}$ and density at $140 \mathrm{~cm}$ from the inlet in Ellison \& Turner's laboratory experiments shows that no shape preserving transition can occur with a loss in energy flux unless $F r>158.8$ and when $q>48.6$, both unrealistically large values: in practice, no shape-preserving transition is possible. (Shape preserving transitions of profiles of velocity given by (2) with $n=3$ and 4 with density given by (3) with $\alpha h=3.5$ have also been examined: the values, $F r_{c}$ at which transitions are possible are summarized in table 3 .) 


$\begin{array}{cccc}U(z) & \rho(z) & F r_{c} & q \\ (2), n=3 & (3), \alpha h=3.5 & 4.11 & 2.86 \\ (2), n=4 & (3), \alpha h=3.5 & 1.75 & 1.58 \\ \text { Figure } 1(a) & \text { Figure } 1(c) & 12.5 & 6.36 \\ \text { Figure 2(130 cm) } & \text { Figure 2(140 cm) } & 158.8 & 48.6\end{array}$

TABLE 3. Values of the critical values, $F r_{c}=U_{\max }^{2} / g \Delta h$, required before shape-preserving transitions can occur in model and observed flows, and the smallest value of the jump amplitude, $q$, at which a transition can occur. The higher values of $\mathrm{Fr}_{c}$ and $q$ found for the observed flow in figure 1 (line 3 of the table) compared to those of the model (line 2) derive from an overestimate using the model values of the change in the flux of kinetic energy (the first term on the right-hand side of (A12)) by about $38 \%$ and an underestimate of the remaining flux changes by $29 \%$, for given $q$.

\subsubsection{Transition to a mixed state}

Shape-preserving transitions of the cascading flow in Lake Geneva appear impossible. Suppose that instead of seeking conditions in which the profile shapes are preserved, we investigate the other extreme, a transition that mixes the velocity and density and makes them both uniform beneath the overlying fluid that is still at rest and of density $\rho_{0}(1-\Delta)$. In this case, we can take $F_{2}\left(z / h_{2}\right)=1$ and $f_{2}\left(z / h_{2}\right)=\delta$. Proceeding as before, figure $5(b)$ shows the curves of constant $F r, E=0$ and $S=1$ in the $(q, P)$-plane. When $F r=3.34$, corresponding to the flow in figure 1 , there are transitions with $E>0$ for small values of $P-1$. All are, however, unstable $(S>1)$ in that the flows downstream of transitions are prone to further shape-preserving transitions without entrainment; with mixing characterized by $P$, the chosen flow downstream of the assumed transition is not unique. In this sense no stable energyloosing transition of the observed flow to a mixed state can occur. A value of $\mathrm{Fr}>3.66$ is required for a stable transition to occur with $q>1.47$.

\subsubsection{Transition to other states}

Transitions of the observed flow to other downstream states have been considered. As an example intermediate between the shape-preserving transition and that to a mixed state, we show in figure $5(c)$ the results of taking a downstream density with a uniform gradient, so $f_{2}\left(z / h_{2}\right)=\delta\left(1-z / h_{2}\right)$, and velocity given by (2) with $n=1$. Transitions with $E>0$ can occur when $F r=3.34$ for values of $q<1.13$ and small $P-1$, but these are unstable $(S>1)$. Only when $F r>F r_{c}=5.90$ can stable transitions occur with $E>0$ and then with $q>2.62$. Once again, no stable transition can occur for the velocity and density profiles of figure $1(a, c)$ when $\mathrm{Fr}=3.34$.

The question then arises: 'Can any downstream profiles of density and velocity be found to which the cascading flow observed in Lake Geneva can change in a stable transition with a reduction in energy flux?' To investigate this, density profiles have been taken with the form

$$
f_{2}\left(z / h_{2}\right)=\delta\left[1-z / h_{2}+\sum_{m=1}^{m=3} a_{m} \sin \left(m \pi z / h_{2}\right)\right],
$$

where coefficients $a_{m}$ are constants with $\left|a_{m}\right| \leqslant 1$ (a linear profile perturbed by a series of sinusoidal harmonics) together with velocity profiles

$$
F_{2}\left(z / h_{2}\right)=\sum_{n=1}^{n=4} b_{n} \chi_{n}
$$



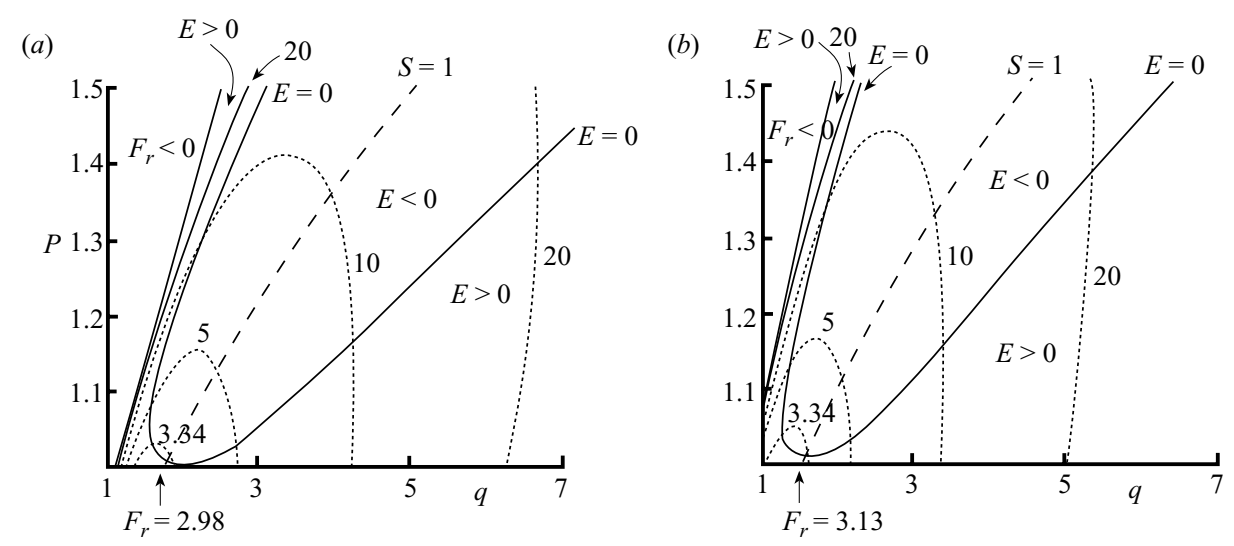

FiguRE 6. The curves corresponding to those in figure when, downstream of a transition, the density profiles are given by (6) and (11) and velocity profiles are either $(a)$, case $(a)$, a sum of terms like those in (2), with $n=1$ to 4 , or $(b)$, case $(b)$, a sum of the first four sinusoidal harmonics. The shapes of the profiles (shown in figure 7) are those for which a stable transition occurs with $E>0$ and with the smallest $F r$.

where $\chi_{n}$ is either $(\mathrm{a}): q_{n}[\pi(1-z / h)]^{n} \sin [\pi(1-z / h)]$ as in (2) but with $q_{n}$ selected to make the maximum value of each term equal to unity, or (b): $\sin \left(n \pi z / h_{2}\right)$ as in (11); the coefficients $b_{n}$ are constants with $\left|b_{n}\right| \leqslant 1$. The coefficients, $a_{m}$ and $b_{n}$, are further confined to values for which the density profiles are statically stable and the velocity is non-negative. For values consistent with this constraint, the smallest value of $F r$ at which $E>0$ and $S<1$ in case $(a)$ is $F r_{c}=2.98$ with $q>1.72$ and in case $(b)$ is $F r_{c}=3.13$ with $q>1.52$. The curves corresponding to those of figure 5 are shown in figure 6.

Since the values of $F r_{c}$ are less than 3.34, the conclusion is that there are downstream flow states to which the flows observed in Lake Geneva may change after a hydraulic jump, although the observed value of $F r$ does not greatly exceed $F r_{c}$, and the flow state in Lake Geneva is, in this sense, marginal. There are only small ranges, $1.9903>q>1.9888$ with corresponding $1 \leqslant P<1.0005$ in case $(a)$, and $1.6149>q>1.6113$ with corresponding $1 \leqslant P<1.0019$ in case $(b)$, in which flows with the observed $\mathrm{Fr}=3.34$ can undergo a stable transition. The density and velocity profiles corresponding to $\mathrm{Fr}_{c}$ for which transitions are possible in cases $(a)$ and $(b)$ are shown in figure 7. (Values of the coefficients, $a_{m}$ and $b_{n}$, giving the smallest value, $\mathrm{Fr}_{c}$, are given in the figure caption.) The most obvious change from the observed profiles (figure $1(a, c)$ is in density where there is a notable increase in the upper part of the cascading water, resulting from mixing within the stratified moving layer.

A further question may be posed: 'If a transition from the observed flow state in Lake Geneva occurs, what is the downstream flow state that is reached with a maximum loss in the energy flux?' We might argue that, if there is in the transition a process that is favoured by greater energy loss, then the most likely flow downstream will be that with the greatest loss in energy flux, or that the most favoured transition is one to a flow state carrying a minimum energy flux downstream. In this case we do not, as above, seek the smallest $F r$, but, setting $F r=3.34$, the flow states in cases $(a)$ and $(b)$ are examined to determine that with the greatest loss in energy flux, insisting that $S<1$, so any transition is stable. The greatest loss, $E=0.027$ (given by 10 ), found is in case $(a)$ when the transition amplitude, $q,=1.88$ and $P=1.0$, so there is no entrainment of the upper layer. The density and velocities downstream of the possible 

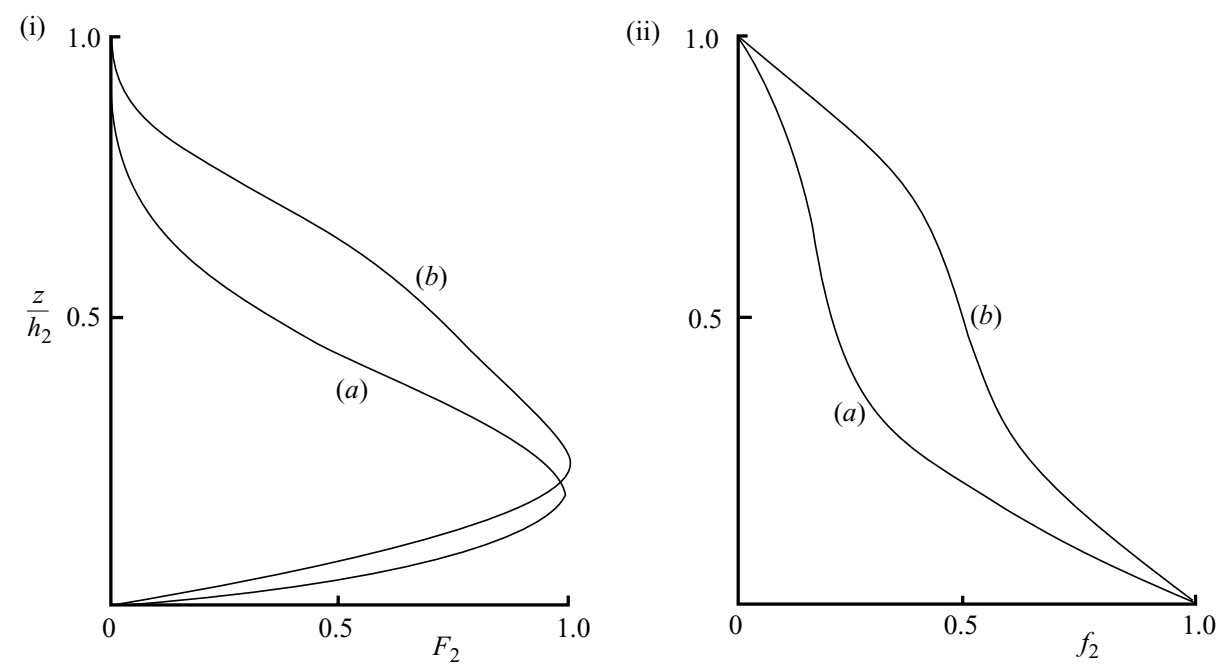

FIGURE 7. The profiles of (i) velocity given by $F_{2}$, and (ii) density given by $f_{2}$ that follow stable transitions with $E>0$ from the cascading flow with the profiles observed in Lake Geneva with minimum $F r$. The two cases, $(a)$ and $(b)$, are as described in the text. The coefficients in (a) are $a_{m}=-0.3,-0.1,0.0$, for $m=1-3$, respectively, and $b_{n} q_{n}=0.0,0.076,0.0,0.0296$, for $n=1-4$, respectively. The coefficients in $(b)$ are $a_{m}=0.0,-0.1,0.0$, for $m=1-3$, respectively, and $b_{n}=0.9,0.4,0.1,0.1$, for $n=1-4$, respectively.
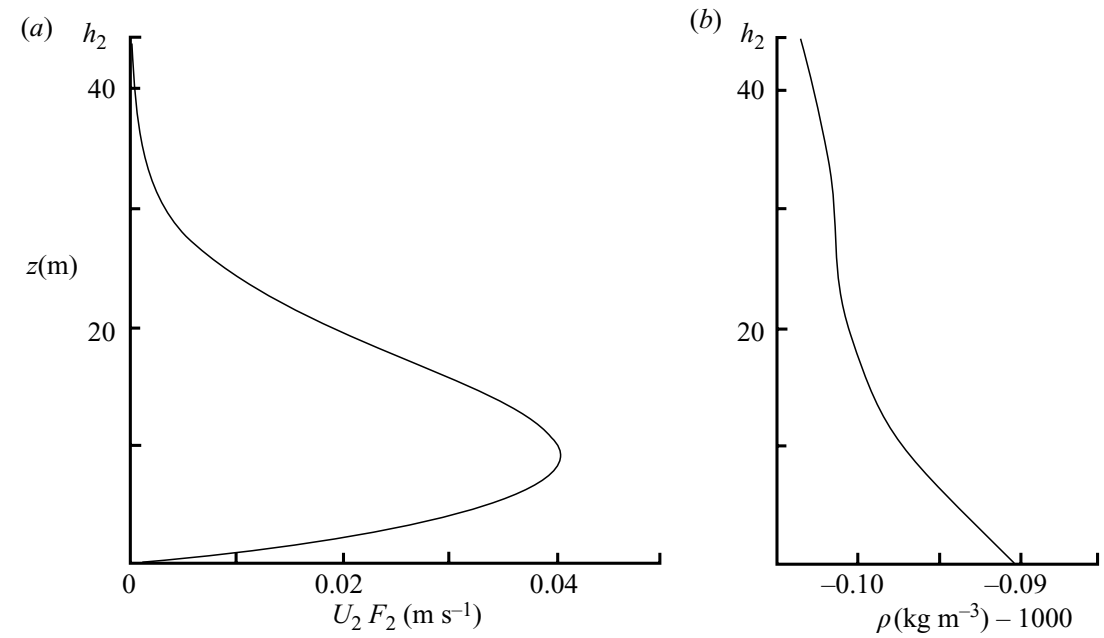

FIGURE 8 . The profiles of $(a)$ down-slope velocity and $(b)$ density that follow stable transitions with the greatest rate of energy loss, $E$, from the density and velocity of the cascading flow observed in Lake Geneva. The profiles are scaled for comparison with the up-stream profiles in figures $(a$ and $c$ ) respectively, from which they have evolved after a transition in which the energy flux is reduced. For comparison with those in figure 7 , the unscaled coefficients are $a_{m}=-0.3,-0.1,0.0$, for $m=1-3$, respectively, and $b_{n} q_{n}=0.0,0.0,0.064,0.0296$, for $n=1-4$, respectively.

transition with maximum loss of energy flux are shown in figure 8, scaled so that they can be compared with the upstream profiles from which they evolve. The coefficients, $a_{m}$ and $b_{n}$, of this solution are given in the figure caption, and differ from those in figure 7, case $(a)$. Both the maximum velocity and the maximum densities are decreased 
in transition whilst the thickness of the flowing and stratified layer is increased by a factor $q$ from $24 \mathrm{~m}$ to $45.1 \mathrm{~m}$. The (dimensional) maximum rate of loss of energy flux per unit width of the cascading flow in a stable transition is $0.027\left[\left(g \Delta h_{1}\right)^{3 / 2} \rho_{0} h_{1}\right]$ or, substituting values, about $6.9 \times 10^{-2} \mathrm{~W} \mathrm{~m}^{-1}$, substantially less than the kinetic energy flux in the cascading flow, $\rho_{0} U_{\max }^{3} h_{1} \int_{0}^{1} F_{1}^{3}(x) \mathrm{d} x / 2$, equal to $1.92 \mathrm{~W} \mathrm{~m}^{-1}$.

\subsubsection{Along-slope flow}

In some limited conditions the effect of an along-slope flow can be taken into account. If, for example, it is supposed that the postulated hydraulic jump is aligned in the $y$-direction (or, in Lake Geneva, that a transition lies along an isobath), the equations of volume and mass flux conservation in the $x$-direction through the transition, (A1) and (A5), are unchanged. So too is that of momentum flux, (A7), since the pressure is hydrostatic. The $y$-component of velocity, taken (in a form similar to that of the $x$-component in (5)) as $V_{i} G\left(z / h_{i}\right)$ in $0<z<h_{i}$, where $V_{i}$ is the maximum $y$-component of the flow, and as $V_{i} G(1)$ in $z>h_{i}$ with $i=1$ and 2 , however, enters into the equation for the rate of loss of energy flux, contributing an additional term

$$
(F r / 2)\left\{\left(V_{1} / U_{1}\right)^{2} \int_{0}^{1} F_{1} G_{1}^{2} \mathrm{~d} x-\left(V_{2} / U_{1}\right)^{2} \int_{0}^{1} F_{2} G_{2}^{2} \mathrm{~d} x\left[\int_{0}^{1} f_{1} F_{1} \mathrm{~d} x / \int_{0}^{1} f_{2} F_{2} \mathrm{~d} x\right]\right\}
$$

to the right-hand side of (A10).

The problem of selecting the flow downstream of the transition remains. If, for example, the transition is shape-preserving with $G_{1}=G_{2}=G$, and if $V_{2} / V_{1}=U_{2} / U_{1}$ whilst selecting $G(1)=0$ (i.e. so that the $y$-axis is chosen to move with the speed of the along-slope flow above the cascading water in Lake Geneva, which does not, however, ensure no-slip at $z=0$ in the $y$-component of flow downstream of the transition) the additional term becomes

$$
(F r / 2)\left(V_{1} / U_{1}\right)^{2} \int_{0}^{1} F G^{2} \mathrm{~d} x\left(1-p^{3} / q^{2}\right) .
$$

Using the values of $V_{1}, U_{1}, F$ and $G$ derived from the data in Lake Geneva (figure $1 a, b$ ), the critical value of $F r$ for a transition to occur is about 5.20 when $P=1$ and $q=3.97$. Although less than that shown in figure $5(a)\left(F r_{c}=12.5\right)$, the critical value still exceeds that observed, 3.34, and no shape-preserving transition appears to be possible.

\section{Discussion}

The short answer to the question 'Are cascading flows stable?' is 'Some (at least) are apparently unstable to some types of disturbance.' The mean flow of the coldwater cascade in Lake Geneva is unstable to Kelvin-Helmholtz instability. The unstable mode 1 disturbances appear to be associated with the shear above the level of the maximum mean down-slope current rather than the near-bed region where turbulence may be sustained by the stress on the bed. The relatively long e-folding time scale of disturbances, about $2 \mathrm{~h}$, in the lake suggests, however, that unstable disturbances will not grow fast enough to control the turbulence within the plume. The results provide support for the conjecture by Turner that flows in inclined plumes are marginally unstable. They are, moreover, consistent with the proposition that some naturallyoccurring, forced turbulent stratified mean shear flows, including perhaps those driven by wind in the upper ocean, are in a similar state of marginal stability.

Whether in cascades there are, as in Mittendorf's experiments, periodic transitions from near laminar flow to turbulent conditions, and back again, is not known. It seems 
unlikely that the finite-amplitude propagating roll waves are related to the marginal stability of the flow. No measurements of turbulence in cascading flows are, however, yet available that might establish, for example, whether turbulence is intermittent or whether the roll waves are more highly turbulent than the flows between.

We have examined the possibility that stationary hydraulic jumps may occur in cascading flows. Some density and velocity profiles are found to which stable transitions are possible. By 'stable' transitions we mean that the flows following transitions are not themselves able to undergo further shape-preserving transitions without entrainment; for given $P$, flows with the chosen profiles are unique. In this restricted and conditional sense, the observed flow appears to be unstable to hydraulic transition; hydraulic jumps may be possible, triggered for example as the flow passes over changes in topography. The smallest values of $F r$ at which transitions $E>0$ and $S<1$ are possible from flows with the density and velocity profiles observed in Lake Geneva, $F r_{c}=2.98$ in case $(a)$ and $F r_{c}=3.13$ in case $(b)(\S 3.2 .3)$, are, however, only slightly less than that of the actual flow. Again, the cascading flow may be regarded as being marginally unstable to transitions.

It was hoped that a study of different downstream states might help in establishing which is most likely. It is of note that the velocity and density profiles, the downstream flow states, found to minimize the value $F r$ (figure 7) and to maximize $E$ at $F r=3.34$ (figure 8) are almost identical, suggesting that a state close to that shown in figure 8 may be favoured. The restricted ranges of possible jump amplitudes, $q$, and entrainment value, $P$, are notable; without some mechanism to enhance the value, $F r$, of the flow (i.e. to increase $U_{\max }$ or to decrease $h_{1}$ ) transitions are only possible within a limited range of jump amplitudes and with moderate entrainment. Without some additional constraint or information about the processes of mixing and their efficiency, or perhaps a condition such as the one of maximum change in energy flux examined in $\S 3.2 .3$, the precise value of jump amplitude or entrainment cannot be determined. It is nevertheless remarkable that the flow state following a possible transition is so severely limited in form and amplitude.

Although solutions are found for stable transitions conserving volume, mass and momentum flux, with energy loss, the existence of stationary transitions at values of $\mathrm{Fr}>\mathrm{Fr}_{c}$ has not been proved and must be in doubt, in spite of the persuasive images of stationary internal hydraulic jumps in the atmosphere, e.g. that in the lee of the Sierra Nevada range illustrated in Turner (1973, figure 3.11) and Lighthill (1978, figure 117) with $q$ possibly in the range 5-10. (Although Turner describes the phenomena as a hydraulic jump, Lighthill is more cautious, calling it 'something like a hydraulic jump'. Scorer (1972) ascribes it to flow separation preceding a rotor in the first of several internal lee waves.) It is usually accepted that transitions occur in flows in which no waves can propagate upstream, carrying energy from a transition and possibly changing the flow approaching a transition. Here however, as found in $\S 2.3 .1$, there are waves that can propagate upstream. The group speed of long internal waves is equal to their phase speed (Thorpe 1978; Baines 1995), implying that these waves may carry energy upstream from the regions of stationary transitions, although only slowly in comparison with $U_{\max }$. Values of $F r>F r_{c}$ may not preclude upstream travelling waves and, in this sense, $F r_{c}$ is not a critical Froude number of the flow. Steady stable and stationary jumps may be possible only in spatially decelerating flows where upstream wave propagation is inhibited, such as radially spreading stratified flows or those in two dimensions over a decreasing bottom slope.

One further point deserves mention. The minimum value of $F r$ found in $\S 3.2 .3$ at which the cascading flow in Lake Geneva can undergo hydraulic jumps, 2.98 in case 
(a) and 3.13 in case $(b)$, are greater than the critical value, $F r_{c}=2.23$, at which the flow becomes unstable to Kelvin-Helmholtz instability. If this instability can occur in a flow as described in $\S 2$, there must be other flow states to which the observed flow will change following instability and the subsequent generation of turbulence and mixing. Rather than produce the overall changes (e.g. in flow depth, $h$ ) found in hydraulic jumps, the instability is instead likely to lead to relatively localized mixing within the initial profiles; such internal mixing may reduce the gradients of density and velocity where instability occurs within the cascading flow, but is unlikely to involve substantial entrainment of the external fluid into the inclined plume or to promote dramatic changes in its structure or thickness, $h$.

Flux conserving, and energy flux reducing, changes of the down-slope velocity and density profiles observed in Lake Geneva have been found in downstream states in which, over limited depth intervals, the density and velocity gradients are reduced or completely destroyed (i.e. the fluid in the latter becoming locally uniform in density and with zero velocity gradient) and in which there is no entrained flux (i.e. $Q=0$ or $P=1$ ) provided the vertical extent of the mixed region is sufficiently small, typically less than $3 \mathrm{~m}$. The absence of conditions in which hydraulic jumps involving entrainment and a substantial change in the density and velocity can occur does not therefore exclude local changes within the interior structure of the cascading water caused by Kelvin-Helmholtz instability.

We are most grateful to Mrs Kate Davis for assistance in the preparation of figures. B. Ozen was funded by the Swiss Science Foundation grant 200020-109349.

\section{Appendix A. Conservation equations in general form}

With velocity, (5), and density, (6), conservation of volume flux from upstream $(i=1)$ to downstream $(i=2)$ through the assumed transition is satisfied by

$$
U_{1} h_{1} \int_{0}^{1} F_{1}(x) \mathrm{d} x+Q=U_{2} h_{2} \int_{0}^{1} F_{2}(x) \mathrm{d} x,
$$

where $Q$ is the flux of fluid of density $\rho_{0}(1-\Delta)$, per unit channel width, from the overlying uniform layer. This can be written

$$
Q /\left(U_{1} h_{1} \int_{0}^{1} F_{1}(x) \mathrm{d} x\right)+1=U_{2} h_{2} \int_{0}^{1} F_{2}(x) \mathrm{d} x /\left(U_{1} h_{1} \int_{0}^{1} F_{1}(x) \mathrm{d} x\right),
$$

or

$$
Q / U_{1} h_{1} \int_{0}^{1} F_{1}(x) \mathrm{d} x=P-1,
$$

where an entrainment factor,

$$
P=U_{2} h_{2} \int_{0}^{1} F_{2}(x) \mathrm{d} x /\left(U_{1} h_{1} \int_{0}^{1} F_{1}(x) \mathrm{d} x\right)>1,
$$

is a measure of the entrainment.

The conservation of mass flux, including the flux $Q \rho_{0}(1-\Delta)$ from the overlying layer, leads in general, using (A1), to a further relation:

$$
U_{1} h_{1} \int_{0}^{1} f_{1}(x) F_{1}(x) \mathrm{d} x=U_{2} h_{2} \int_{0}^{1} f_{2}(x) F_{2}(x) \mathrm{d} x,
$$


so that (A4) can be written

$$
P=\int_{0}^{1} f_{1}(x) F_{1}(x) \mathrm{d} x \int_{0}^{1} F_{2}(x) \mathrm{d} x /\left(\int_{0}^{1} f_{2}(x) F_{2}(x) \mathrm{d} x \int_{0}^{1} F_{1}(x) \mathrm{d} x\right) .
$$

Momentum conservation (conservation of $\int p_{i} \mathrm{~d} z+\int\left(\rho_{i} U_{i}\right) U_{i} \mathrm{~d} z$, where $p_{i}$ is the pressure, assumed to be hydrostatic upstream and downstream of a transition) leads to

$$
\begin{aligned}
U_{2}^{2} h_{2} \int_{0}^{1} F_{2}^{2}(x) \mathrm{d} x & -U_{1}^{2} h_{1} \int_{0}^{1} F_{1}^{2}(x) \mathrm{d} x \\
& =2 g \Delta\left[h_{1}^{2} \int_{0}^{1} \int_{x}^{1} f_{1}(y) \mathrm{d} y \mathrm{~d} x-h_{2}^{2} \int_{0}^{1} \int_{x}^{1} f_{2}(y) \mathrm{d} y \mathrm{~d} x\right],
\end{aligned}
$$

where $g$ is the acceleration due to gravity and $\Delta \ll 1$. Writing $q=h_{2} / h_{1}$ and, using (A5),

$$
U_{2}=U_{1} \int_{0}^{1} f_{1}(x) F_{1}(x) \mathrm{d} x /\left(q \int_{0}^{1} f_{2}(x) F_{2}(x) \mathrm{d} x\right)
$$

(A7) gives

$$
\begin{aligned}
U_{1}^{2} / g \Delta h_{1}= & 2 q\left[q^{2} \int_{0}^{1} \int_{x}^{1} f_{2}(y) \mathrm{dy} \mathrm{d} x-\int_{0}^{1} \int_{x}^{1} f_{1}(y) \mathrm{d} y \mathrm{~d} x\right] / \\
& {\left[\int _ { 0 } ^ { 1 } F _ { 1 } ^ { 2 } ( x ) \mathrm { d } x \left\{q-P^{2} \int_{0}^{1} F_{2}^{2}(x) \mathrm{d} x\left(\int_{0}^{1} F_{1}(x) \mathrm{d} x\right)^{2} /\right.\right.} \\
& {\left.\left.\left[\int_{0}^{1} F_{1}^{2}(x) \mathrm{d} x\left(\int_{0}^{1} F_{2}(x) \mathrm{d} x\right)^{2}\right]\right\}\right] }
\end{aligned}
$$

as a condition for a transition to occur.

In general, a non-dimensional loss in energy flux per unit channel width, $E^{\prime}$ (the change in the sum of the kinetic energy flux, $\int\left(\rho_{i} U_{i}^{2} / 2\right) U_{i} \mathrm{~d} z$, and the potential energy flux, $\int g \rho_{i} z U_{i} \mathrm{~d} z$, accounting for the work done by the pressure force, $\int p_{i} U_{i} \mathrm{~d} z$ ) can be written as

$$
\begin{aligned}
E^{\prime} /\left(g \rho_{0} \Delta U_{1} h_{1}^{2}\right) \\
\left.=\left(U_{1}^{2} / 2 g \Delta h_{1}\right)\left\{\int_{0}^{1} F_{1}^{3}(x) \mathrm{d} x-\int_{0}^{1} F_{2}^{3}(x) \mathrm{d} x\left[\int_{0}^{1} f_{1}(x) F_{1}(x) \mathrm{d} x / \int_{0}^{1} f_{2}(x) F_{2}(x) \mathrm{d} x\right]\right]^{3} / q^{2}\right\} \\
+2\left[\int_{0}^{1} x F_{1}(x) \mathrm{d} x-\int_{0}^{1} x f_{1}(x) F_{1}(x) \mathrm{d} x+\int_{0}^{1} F_{1}(x) \int_{x}^{1} f_{1}(y) \mathrm{d} y \mathrm{~d} x\right] \\
-2 q\left[\int_{0}^{1} f_{1}(x) F_{1}(x) \mathrm{d} x / \int_{0}^{1} f_{2}(x) F_{2}(x) \mathrm{d} x\right] \\
\quad \times\left[\int_{0}^{1} x F_{2}(x) \mathrm{d} x-\int_{0}^{1} x f_{2}(x) F_{2}(x) \mathrm{d} x+\int_{0}^{1} F_{2}(x) \int_{x}^{1} f_{2}(y) \mathrm{d} y \mathrm{~d} x\right],
\end{aligned}
$$

provided that the kinetic energy flux carried by the entrained flow is negligible, with $U_{1}^{2} / g \Delta h_{1}$ given by (A9). With no source of energy flux within the transition, $E^{\prime}$ must not be negative if the transition is physically possible. 
The flow downstream of a transition may be regarded as unstable and, with the chosen profiles of density and velocity, is not unique if a further transition is possible with no entrainment $(P=1)$ to a flow with the same velocity and density profiles. Proceeding as in Thorpe (2007), we find that the condition for instability is

$$
\begin{aligned}
& U_{1}^{2} / g \Delta h_{1} \\
& >4 q^{3}\left[\int_{0}^{1} \int_{x}^{1} f_{2}(y) \mathrm{d} y \mathrm{~d} x / \int_{0}^{1} F_{2}^{2}(x) \mathrm{d} x\right] \\
& \quad \times\left[\int_{0}^{1} f_{2}(x) F_{2}(x) \mathrm{d} x / \int_{0}^{1} f_{1}(x) F_{1}(x) \mathrm{d} x\right]^{2} f_{2}(0) .
\end{aligned}
$$

Noting that $F r=U_{1}^{2} / g \Delta h_{1}$, we define a stability parameter $S$ as the ratio of the left-hand side of to the right-hand side of (A11):

$$
\begin{aligned}
S \equiv & F r /\left\{4 q^{3}\left[\int_{0}^{1} \int_{x}^{1} f_{2}(y) \mathrm{d} y \mathrm{~d} x / \int_{0}^{1} F_{2}^{2}(x) \mathrm{d} x\right]\right. \\
& \left.\times\left[\int_{0}^{1} f_{2}(x) F_{2}(x) \mathrm{d} x / \int_{0}^{1} f_{1}(x) F_{1}(x) \mathrm{d} x\right]^{2} f_{2}(0)\right\},
\end{aligned}
$$

where $S>1$ implies instability and $S<1$ implies stability, when no further shapepreserving transition can occur in the flow downstream of the transition without entrainment from the upper layer. Two conditions are therefore applied for transitions to be possible, $E^{\prime} \geqslant 0$ and $S>1$.

For the shape-preserving transitions with $F_{1}\left(z / h_{1}\right)=F_{2}\left(z / h_{2}\right),=F(x)$, say, and $f_{1}\left(z / h_{1}\right)=f(x), f_{2}\left(z / h_{2}\right)=\delta f(x)$, so that $f_{2}(0)=\delta$ because $f_{1}(0)=1$, (A12) reduces to

$$
S=F r /\left\{4 q^{3}\left[\int_{0}^{1} \int_{x}^{1} f(y) \mathrm{d} y \mathrm{~d} x / \int_{0}^{1} F^{2}(x) \mathrm{d} x\right] \delta^{4}\right\} .
$$

Since in this case $P=1 / \delta$ from (A6), and (A9) gives

$$
F r=U_{1}^{2} / g \Delta h_{1}=2 q\left(q^{2}-P\right) \int_{0}^{1} \int_{x}^{1} f(y) \mathrm{d} y \mathrm{~d} x /\left[P\left(q-P^{2}\right) \int_{0}^{1} F^{2}(x) \mathrm{d} x\right],
$$

(A13) becomes

$$
S=P^{3}\left(q^{2}-P\right) /\left[2 q^{2}\left(q-P^{2}\right)\right]>1,
$$

for instability to occur. (This is independent of the form of the profiles of density and velocity and so the curve, $S=1$ in figure $5(a)$ is valid for all shape-preserving transitions.)

\section{REFERENCES}

BaInes, P. G. 1995 Topographic Effects in Stratified Flows. Cambridge University Press.

BELL, T. H. 1974 Effects of shear on the properties of internal gravity waves. Deutsche Hydrograph. Z. 27, 57-62.

Benjamin, T. B. \& Lighthill, M. J. 1954 On cnoidal waves and bores. Proc. R. Soc. Lond. A 224, $448-460$.

Cenedese, C., Whitehead, J. A., Ascarelli, T. A. \& Ohiwa, M. 2004 A dense current flowing down a sloping bottom in a rotating fluid. J. Phys. Oceanogr. 34, 188-203. 
Drazin, P. G. \& ReID, W. H. 1981 Hydrodynamic Stability. Cambridge University Press.

Ellison, T. H. \& Turner, J. S. 1959 Turbulent entrainment in stratified fluids. J. Fluid Mech. 6, 423-448.

Fer, I., Lemmin, U. \& ThORPe, S. A. $2002 a$ Winter cascading of cold water in Lake Geneva. J. Geophys. Res. 107, C6, 10.1029/2001JC000828, 13-1-13-16.

Fer, I., Lemmin, U. \& Thorpe, S. A. $2002 b$ Contribution of entrainment and vertical plumes to the winter cascading of cold waters in a deep lake. Limnol. Oceanogr. 47, 576-580.

GASTER, M. 1962 A note on the relation between temporally-increasing and spatially-increasing disturbances in an infinitely deep, continuously stratified fluid. J. Fluid Mech. 14, 222-224.

Gray, T. E., Alexander, J. \& Leeder, M. R. 2006 Longitudinal flow evolution and turbulence structure of dynamically similar, saline density and turbidity currents. J. Geophys. Res. 111, C08015, doi:10.1029/2005JC003089.

Hazel, P. 1972 Numerical studies of the stability of inviscid stratified shear flows. J. Fluid Mech. 51, 39-61.

Holland, D. M., Rosales, R. R., Stefanica, D. \& Tabak, E. G. 2002. Internal hydraulic jumps and mixing in two-layer flows. J. Fluid Mech. 470, 63-83.

Lighthill, J. 1978 Waves in Fluids. Cambridge University Press.

Miles, J. W. 1967 Internal waves in a continuously stratified atmosphere or ocean. J. Fluid Mech. 28, 305-310.

Mittendorf, G. H. 1961 The instability of stratified flow. MSc thesis. State University of Iowa.

Neiman, P. J., Hardesty, R. M., Shapiro, M. A. \& Cupp, R. E. 1988 Doppler lidar observations of a downslope windstorm. Mon. Weather Rev. 116, 2265-2275.

PaWlaK, G. \& Armi, L. 2000 Mixing and entrainment in developing stratified currents. J. Fluid Mech. 424, 45-73.

Peltier, W. R. \& Scinocca, J. F. 1990 The origin of severe downslope windstorm pulsations. J. Atmos. Sci. 47, 2853-2870.

Pratt, L., Deese, H., Murray, S. \& Johns, W. 2000 Continuous dynamical modes in straits having arbitrary cross sections, with applications to the Bab el Mandab. J. Phys. Oceanogr. 30, $2515-2534$.

Press, W. H., Teukolski, S. A., Vetterling, W. T. \& Flannery, B.P. 1992 Numerical Recipes in C: the Art of Scientific Computing, 2nd edn. CambridgeUniversity Press.

SCORER, R. 1972 Clouds of the World. Lothian, Melbourne.

Simpson, J. E. 1997 Gravity currents. 2nd edn. Cambridge University Press.

THORPE, S. A. 1978 On the shape and breaking of finite amplitude internal gravity waves in a shear flow. J. Fluid Mech. 85, 7-31.

Thorpe, S. A. 1999 A note on the breaking of internal waves in the ocean. J. Phys. Oceanogr. 29, 2433-2441.

ThORPE, S. A. 2007 Dissipation in hydraulic transitions in flows through abyssal channels. J. Mar. Res.

Thorpe, S. A. \& Hall, A. J. 1977 Mixing in upper layer of lake during heating cycle. Nature, 265, $719-722$.

Turner, J. S. 1973 Buoyancy Effects in Fluids. Cambridge University Press. 